\title{
MDS-based Localization Algorithm for RFID Systems
}

\author{
Wenbo Shi and Vincent W.S. Wong \\ Department of Electrical and Computer Engineering, \\ The University of British Columbia, Vancouver, Canada \\ E-mail: \{wenbos, vincentw $\} @$ ece.ubc.ca
}

\begin{abstract}
In radio frequency identification (RFID) systems, location information is of great importance to provide locationaware services combined with identification. Conventional RFID systems can only provide coarse localization information. In this paper, we propose a novel approach named MDS-RFID to locate active RFID tags based on multidimensional scaling (MDS), an efficient data analysis technique. The approach has the advantage of fully utilizing the distance information in the network simultaneously, and thus can achieve better localization results than previous multilateration-based methods. The MDSRFID algorithm first infers the tag-to-reader distances from the received signal strength (RSS). To obtain the distance matrix, the inter-tag distances are estimated using a triangular method. Then, classical MDS algorithms can be applied to determine the estimated locations of the tags. An optional refinement step can be added to further improve the accuracy using maximum likelihood estimation at the expense of additional computation costs. Simulation results show that the MDS-RFID algorithm can achieve a significant gain in accuracy over the previous localization schemes based on multilateration using only a few readers.
\end{abstract}

\section{INTRODUCTION}

Radio frequency identification (RFID) is a wireless technology that enables data communications via radio waves between a reader and a tag which is attached to an object for identification and tracking [1]. An RFID system is composed of RFID tags, readers, and servers. An RFID tag is a microchip combined with an antenna. It is able to store the object information that can be retrieved by the reader via radio waves, which provides unique identification for every object in the system.The reader has an interface to communicate with the server, sending the data it receives from the tags to the server via wired or wireless communications. RFID systems can be enhanced greatly if the identification information is combined with locations to provide many attracting locationaware applications (e.g., mobility control, resource allocation, security, and service discovery).

Several RFID localization schemes have been proposed in the literature. They can be classified into: distance estimation, scene analysis, and proximity [2]. The algorithms based on distance estimation use multilateration to estimate the positions of RFID tags. The ranging techniques include received signal strength (RSS) [3], time of arrival (TOA) [4], time difference of arrival (TDOA) [5], and received signal phase (RSP) [6]. Scenes analysis approaches use k-nearest-neighbor (kNN) [7] or probabilistic methods [8], [9] to locate RFID tags by matching the measurements to the fingerprints collected from the environment. Proximity is another group of methods to locate RFID tags assuming that the position of the target tag is the same with the reader which detects the tag. Based on the type of RFID tags used in the system, the localization schemes can be classified as being active [3], [7] or passive [10], [11] RFID tag localization as well. An active RFID tag powered by a cell battery has a much longer communications range than a passive tag. In this work, we mainly focus on active RFID tag localization.

SpotON [3] is a RFID location sensing system which belongs to the family of distance estimation. SpotON uses RSS measurements from long range active RFID tags to approximate the distances between tags and readers. Multiple readers collect the RSS measurements and then transform the RSS measurements into distance estimations through a function defined with empirical data. Multilateration is then applied to calculate the positions of the tags.

LANDMARC [7] is an active RFID locating system using scene analysis. It requires reference tags to be deployed at known positions. RSS measurements from multiple readers are used to locate the target RFID tag using the locations of the $k$ nearest reference tags. Different weights are imposed on the $k$ nearest reference tags to estimate the location of the target tag. In the LANDMARC system, dense deployment of reference tags are necessary for good performances. Several extensions based on the LANDMARC system have also been proposed in the literature [12], [13].

In the context of localization, multidimensional scaling (MDS) [14] can be used to solve the localization problem efficiently where the similarity measures in MDS correspond to the Euclidean distances. MDS-based localization algorithm (MDS-MAP) has been well studied in wireless sensor networks [15]-[17]. The MDS-MAP algorithm first starts with forming the distance matrix by running a shortestpath algorithm. Next, MDS is performed to determine the estimated locations of the sensors that best fit those distance measurements. Finally, anchors are used to calculate the absolute coordinates based on linear transformations and rotations. MDS-MAP is a popular localization algorithm in wireless sensor networks and it has many variants (e.g., MDS$\operatorname{MAP}(C)$ [15] which uses metric MDS, MDS-MAP(O) [18] which adopts ordinal MDS, and MDS-MAP(P) [17] which is a distributed scheme using patches of relative map). Additional 
refinement can be added to MDS-MAP based on least-squares minimization to better match the measured distances [17].

MDS has good potentials to solve the localization problem in RFID systems. MDS is more robust to range errors [17]. In addition, MDS does not need many anchors (readers in RFID systems) for localization [15], which is cost-effective. This is because the classical MDS is performed without using the anchors, the anchor information is only used in the last step to obtain the absolute coordinates when the overall structure between nodes have already been determined.

Unfortunately, MDS cannot be applied directly to solve the tag localization problem in RFID system. MDS requires the distance matrix which describes the pairwise distances in the network. However, due to the characteristics of RFID systems, only the tag-to-reader distances can be obtained from the RSS measurements while the inter-tag distances are missing. In order to apply MDS to RFID localization, the primary task is to obtain the inter-tag distance information from the tag-toreader RSS measurements.

In this paper, we study the problem of locating active RFID tags. We propose a MDS-based localization algorithm named MDS-RFID to solve the problem. The contributions of this paper can be summarized as follows:

- We propose to use MDS for active tag localization in RFID systems. A key advantage of the MDS-RFID algorithm over existing schemes is that it can locate all the tags simultaneously using all the distance information in the network so that it can better tolerate noises.

- We add an optional refinement step which solves a maximum likelihood estimation (MLE) problem to further reduce localization errors when enough RFID readers are deployed. One important advantage of the MDS-RFID algorithm is that MDS can provide better initial location estimates than other methods for the refinement.

- We conduct extensive simulations to evaluate the performance of the proposed MDS-RFID algorithm. Simulation results show that the MDS-RFID algorithm outperforms the conventional multilateration-based algorithm using RSS [3] by a considerable margin.

The rest of this paper is organized as follows: In Section II, we describe our proposed method to construct the distance matrix. We present our proposed MDS-RFID algorithm. In Section III, we evaluate the performance of our proposed MDSRFID algorithm and compare it with existing multilaterationbased method [3]. Conclusions are given in Section IV.

\section{The MDS-RFID ALgORITHM}

The system consists of a server, some readers, many active RFID tags. The readers are deployed at locations which are pre-determined. They act as anchors in the network. The RFID tags are attached on the objects which need to be located. We assume that the readers are all within the communications range of the tags, so that each reader can measure the RSS from all the tags. This assumption can be relaxed for large scale networks by dividing into small sub-networks. The RSS from the RFID tag in the system is measured at each reader in
dB. All of the RSS measurements are aggregated to the server where the MDS-RFID localization algorithm is performed. The objective of the localization algorithm is to estimate the positions of the RFID tags given all the RSS measurements in the system. The application environment is typically indoor environment. The MDS-RFID algorithm consists of three steps: data pre-processing step, multidimensional scaling (MDS), and an optional refinement step.

\section{A. Data Pre-processing Step}

The first step of the MDS-RFID algorithm is to construct the distance matrix as the input for MDS. The data available in the RFID system are the RSS measurements from all the tags reported at each reader. The data pre-processing step has two objectives: to infer the tag-to-reader distances from the RSS measurements and to estimate the inter-tag distances based on the tag-to-reader distance information. The distance matrix which describes the measured distances between all pairwise tags and readers is available after the data pre-processing step.

1) RSS-distance Model: To infer the distance between a transmitter (i.e., active RFID tag) and a receiver (i.e., reader) from the RSS, we need to model the path loss which describes the relationship between the signal attenuation and the distance. We use the following log-normal distance path loss model for indoor environment [19]:

$$
P L(d)=P L\left(d_{0}\right)+10 n \log \left(\frac{d}{d_{0}}\right)+z,
$$

where $P L(d)$ is a random variable describing the path loss measured in $\mathrm{dB}$ at distance $d, d_{0}$ is the reference distance, $n$ is the path loss exponent, and $z$ is a random variable which follows a Gaussian distribution $\mathcal{N}\left(0, \sigma^{2}\right)$. The path loss exponent $n$ is equal to 2 in free space and it ranges from 2 to 4 in indoor environment. The standard deviation $\sigma$ in the model usually fluctuates between $3 \mathrm{~dB}$ and $12 \mathrm{~dB}$ in indoor environment [19].

Given the transmission power $P_{t}$, the transmitter antenna gain $G_{t}$, and the receiver antenna gain $G_{r}$, the RSS at distance $d, P(d)$ (in $\mathrm{dB}$ ) can be obtained as

$$
\begin{aligned}
P(d) & =P_{t}+G_{r}+G_{t}-P L(d) \\
& =P_{t}+G_{r}+G_{t}-P L\left(d_{0}\right)-10 n \log \left(\frac{d}{d_{0}}\right)-z \\
& =c-10 n \log (d)-z,
\end{aligned}
$$

where $c=P_{t}+G_{r}+G_{t}-P L\left(d_{0}\right)+10 n \log \left(d_{0}\right)$ is a constant.

From (2), we can obtain the RSS-distance model to estimate the distance $d$ from the measured RSS $P$ (in $\mathrm{dB}$ ) as

$$
\hat{d}(P)=10^{\frac{c-P}{10 n}} .
$$

Note that in a real environment, the RSS may not follow the model exactly due to multipath propagations and some other factors. However, it can be shown in experiments that MDS is able to tolerate such inaccuracies. 


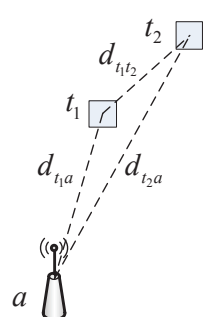

(a)

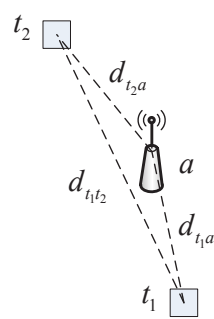

(b)
Fig. 1. (a) Lower bound of $d_{t_{1} t_{2}}$ is the case when tags $t_{1}$ and $t_{2}$ are on the same side of the reader $a$. (b) Upper bound of $d_{t_{1} t_{2}}$ is the case when reader $a$ lies in the middle of tags $t_{1}$ and $t_{2}$.

2) Inter-tag Distance Estimation: Let $\mathcal{A}$ denote the set of readers and $\mathcal{T}$ denote the set of active RFID tags. We denote the tag-to-reader distance between reader $a \in \mathcal{A}$ and tag $t \in \mathcal{T}$ to be $d_{t a}$. Given the RSS measurement $P_{t a}$ from tag $t$ at reader $a$, the tag-to-reader distance $d_{t a}$ can be estimated using (3)

$$
\hat{d}_{t a}=10^{\frac{c-P_{t a}}{10 n}} \text {. }
$$

However, since a tag cannot communicate with another tag, we cannot use the same method to estimate the intertag distance. To solve this problem, we propose a triangular method using the triangle formed by the two tags $t_{i}, t_{j}$, and the reader $a$ to estimate the inter-tag distance between tag $t_{i}$ and $t_{j}$. Given the true tag-to-reader distances $d_{t_{i} a}$ and $d_{t_{j} a}$, the triangular inequalities can be used to give an upper bound and a lower bound of the inter-tag distance $d_{t_{i} t_{j}}$

$$
\left|d_{t_{i} a}-d_{t_{j} a}\right| \leq d_{t_{i} t_{j}} \leq d_{t_{i} a}+d_{t_{j} a}
$$

An illustration of the lower bound and the upper bound of the inter-tag distance is shown in Fig. 1. Two tags $t_{1}, t_{2}$ and one reader $a$ are deployed. It can be seen that the inter-tag distance $d_{t_{1} t_{2}}$ can be estimated by the lower bound $\left|d_{t_{1} a}-d_{t_{2} a}\right|$ when the two tags are placed on the same side of the reader. The upper bound of the distance between $t_{1}$ and $t_{2}$ corresponds to the scenario when the reader lies in the middle of the tags.

The bounds of the inter-tag distance are tight when there are more readers available in the system as we can use more triangles formed by the two tags and the readers. The inter-tag distance can then be estimated by the maximum lower bound and the minimum upper bound of (5) using all the readers.

So far, we only consider the estimation of the inter-tag distance using the true tag-to-reader distances $d_{t a}$. If we use $\hat{d}_{t a}$ which includes measurement errors, the inter-tag distance can be better estimated by averaging the bounds given by the triangular method using all readers:

$$
d_{t_{i} t_{j}}^{l}=\frac{\sum_{a \in \mathcal{A}}\left(\left|\hat{d}_{t_{i} a}-\hat{d}_{t_{j} a}\right|\right)}{|\mathcal{A}|}
$$

and

$$
d_{t_{i} t_{j}}^{u}=\frac{\sum_{a \in \mathcal{A}}\left(\hat{d}_{t_{i} a}+\hat{d}_{t_{j} a}\right)}{|\mathcal{A}|}
$$

where $d_{t_{i} t_{j}}^{l}$ and $d_{t_{i} t_{j}}^{u}$ are the lower bound and the upper bound of the inter-tag distance $d_{t_{i} t_{j}}$ using estimated tag-to-reader distances, respectively, and $|\mathcal{A}|$ is the number of readers.

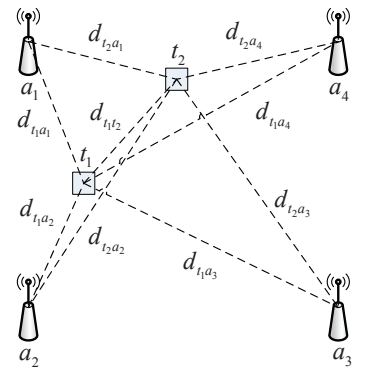

Fig. 2. Estimation of the inter-tag distance $d_{t_{1} t_{2}}$ when readers are deployed at corners of the area.

When the readers are placed at corners of the deployment area, it is impossible for the readers to lie in the middle of two tags. Only the lower bound is effective to estimate the inter-tag distance. In this case, the distance $d_{t_{i} t_{j}}$ can be estimated by the lower bound $\hat{d}_{t_{i} t_{j}}=d_{t_{i} t_{j}}^{l}$. If readers are deployed randomly, the distance $d_{t_{i} t_{j}}$ can be estimated by $\hat{d}_{t_{i} t_{j}}=\left(d_{t_{i} t_{j}}^{l}+d_{t_{i} t_{j}}^{u}\right) / 2$, since it is unknown which bound is more effective.

An example of the inter-tag distance estimation when the readers are deployed at the corners of the area is shown in Fig. 2. Four readers $a_{1}, a_{2}, a_{3}, a_{4}$ are placed at the corners of the deployment area. Two tags $t_{1}, t_{2}$ need to be located. Given all the tag-to-reader distances, it can be seen that the inter-tag distance between $t_{1}$ and $t_{2}$ can best be estimated by the lower bound $\left|d_{t_{2} a_{2}}-d_{t_{1} a_{2}}\right|$ if the distance measurements are accurate. In the presence of measurement errors, equation (6) would produce a better estimate of the inter-tag distance.

\section{B. Multidimensional Scaling (MDS)}

In the MDS-RFID algorithm, we use the classical metric MDS [20]. The similarity measures in MDS are treated as the distances in the Euclidean space. The objective of MDS is to find a placement of points in multidimensional space as a best fit to the similarity measures by a linear transformation. Here, we do not differ the readers and RFID tags. Suppose there are $m$ points with positions $\mathbf{x}_{i}, i=1,2, \ldots, m$. Let matrix $X=\left[\begin{array}{llll}\mathbf{x}_{1} & \mathbf{x}_{2} & \ldots & \mathbf{x}_{m}\end{array}\right]$. The dimension of $X$ is $2 \times m$, where we consider the localization problem to be 2dimensional. Let $D=\left[\hat{d}_{i j}\right]$ denote the matrix of pairwise distance measurements, where $\hat{d}_{i j}$ corresponds to the measured distance between points $\mathbf{x}_{i}$ and $\mathbf{x}_{j}$ for $i \neq j$, and $\hat{d}_{i i}=0$ for all $i$. The goal of MDS is to find an assignment of $X$ in 2dimensional space that minimizes a stress function

$$
\hat{X}=\operatorname{argmin}_{X} \operatorname{Stress}(X),
$$

where the stress function measuring the goodness of fit is defined as [14]

$$
\operatorname{Stress}(X)=\sqrt{\frac{\sum\left(\hat{d}_{i j}-\left\|\mathbf{x}_{i}-\mathbf{x}_{j}\right\|\right)^{2}}{\sum\left\|\mathbf{x}_{i}-\mathbf{x}_{j}\right\|^{2}}},
$$

where $\left\|\mathbf{x}_{i}-\mathbf{x}_{j}\right\|$ is the Euclidean distance between $\mathbf{x}_{i}$ and $\mathbf{x}_{j}$.

In classical MDS, the elements of $\hat{X}$ can be computed using eigenvalue decomposition (EVD) of the double centered squared distance matrix. The squared distance matrix $D^{2}$ is 
defined as $\left[\hat{d}_{i j}^{2}\right]$. The elements of the double centered squared distance matrix $B_{m \times m}$ are determined as [14]

$$
\begin{aligned}
b_{i j} & =-\frac{1}{2}\left(\hat{d}_{i j}^{2}-\frac{1}{m} \sum_{k=1}^{m} \hat{d}_{k j}^{2}-\frac{1}{m} \sum_{k=1}^{m} \hat{d}_{i k}^{2}+\frac{1}{m^{2}} \sum_{k=1}^{m} \sum_{l=1}^{m} \hat{d}_{k l}^{2}\right) \\
& =\hat{\mathbf{x}}_{i}^{T} \hat{\mathbf{x}}_{j} .
\end{aligned}
$$

Note that $B$ is a positive definite matrix having $m$ positive eigenvalues [14]. We can obtain $\hat{X}$ using $U$ and $V$ which are the results of performing EVD to the matrix $B$ [21]:

$$
\begin{aligned}
& B=U V U^{T}=\hat{X}^{T} \hat{X}, \\
& \hat{X}=U V^{1 / 2} .
\end{aligned}
$$

The elements of the solution $\hat{X}$ from (10) are in $m$ dimensional space. To reduce it to 2-dimensional space, we only keep the two largest eigenvalues from $V$ and the corresponding eigenvectors. Now we have the proper dimension of $\hat{\mathbf{x}}_{i}$, but they are in a relative coordinate. To determine the absolute coordinate, the locations of the readers are needed to find an optimal transformation using the coordinate system registration algorithm [22]. Note that we only present the classical MDS here, it is possible to apply more advanced MDS algorithms with higher computation costs. If classical MDS is adopted, we name the algorithm as MDS-RFID(C) where $\mathrm{C}$ stands for classical.

\section{Refinement}

The positions yielded by MDS in the last section can further be refined using maximum likelihood estimation (MLE). Recall from Section II-A, we use the log-normal path loss model to estimate the tag-to-reader distance from the RSS measurement. The tag-to-reader distance estimate $\hat{d}_{t a}$ defined by (3) is a random variable of the true distance $d_{t a}$ :

$$
\begin{aligned}
\hat{d_{t a}} & =10^{\frac{c-P t a}{10 n}} \\
& =d_{t a} 10^{\frac{z}{10 n}} .
\end{aligned}
$$

Then, we can determine the probability distribution function (PDF) of $\hat{d}_{t a}$ as

$$
f\left(\hat{d_{t a}}\right)=\frac{10 n}{\hat{d_{t a}} \ln 10 \sqrt{2 \pi} \sigma} e^{-\frac{\left(10 n \log \left(\hat{d}_{t a} / d_{t a}\right)\right)^{2}}{2 \sigma^{2}}} .
$$

Assuming that the distance measurements from different readers are independent, the PDF of the observation $\left(\hat{d}_{t a_{1}}, \hat{d}_{t a_{2}}, \ldots, \hat{d}_{t a_{|\mathcal{A}|}}\right)$ can be calculated as

$$
\begin{aligned}
f\left(\hat{d}_{t a_{1}}, \hat{d}_{t a_{2}}, \ldots, \hat{d}_{t a_{|\mathcal{A}|}}\right) & =\prod_{i=1}^{|\mathcal{A}|} f\left(\hat{d}_{t a_{i}}\right) \\
& =\alpha e^{\sum_{i=1}^{|\mathcal{A}|}-\frac{\left(10 n \log \left(\hat{t}_{t a_{i}} / d_{t a_{i}}\right)\right)^{2}}{2 \sigma^{2}}}
\end{aligned}
$$

where $\alpha$ is a constant, $\sigma$ is the standard deviation of the noise, and $n$ is the path loss exponent.

MLE produces the estimation of the parameters that maximizes the probability of the observation. Based on the PDF
Algorithm 1 - MDS-RFID Algorithm: Executed by the RFID system server.

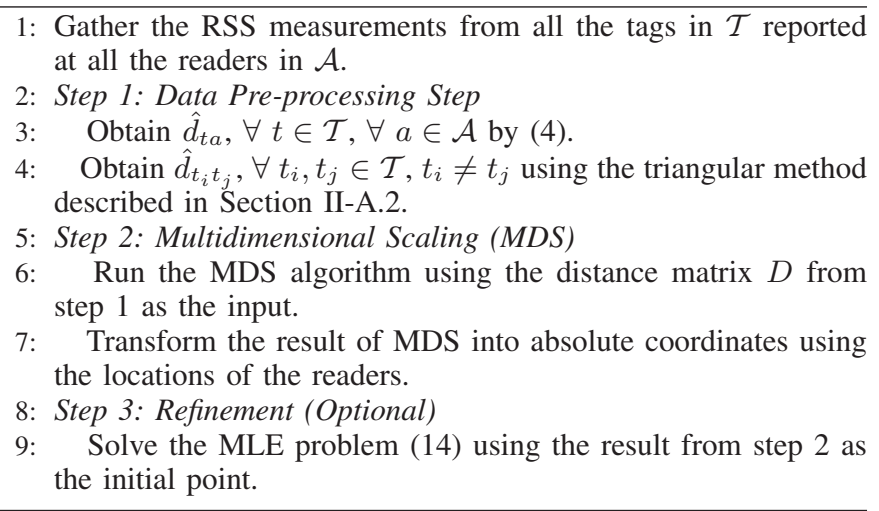

of the observation (13), we can devise the MLE for the MDSRFID algorithm as

$$
\begin{aligned}
\hat{\mathbf{x}}_{t} & =\operatorname{argmax}_{\mathbf{x}_{t}} f\left(\hat{d}_{t a_{1}}, \hat{d}_{t a_{2}}, \ldots, \hat{d}_{t a_{|\mathcal{A}|}}\right) \\
& =\operatorname{argmin}_{\mathbf{x}_{t}} \sum_{i=1}^{|\mathcal{A}|} \frac{\left(10 n \log \left(\hat{d}_{t a_{i}} / d_{t a_{i}}\right)\right)^{2}}{2 \sigma^{2}},
\end{aligned}
$$

where $d_{t a_{i}}=\left\|\mathbf{x}_{t}-\mathbf{x}_{a_{i}}\right\|$.

The problem (14) is a nonlinear optimization problem which does not have a global optimal value. However, it can be solved using some local search techniques providing a good initial point. Using randomly generated initial points will reach some local minima far away from the true positions. One important advantage of the MDS-RFID algorithm is that the coarse positions estimated by MDS can provide a better initial solution for the refinement than other multilaterationbased or heuristic methods. We call the scheme MDS-RFID $(C$, $\mathrm{R})$ if the refinement is added at the expense of additional computations. Note that solving the MLE problem above does not always improve the localization results produced by MDS. If there are only a few readers available in the RFID system, MDS which utilizes all the distances can generate a better topology than the refinement using only the tag-to-reader distance measurements. As the number of readers increases, adding the refinement step will show its advantage.

A complete description of the MDS-RFID algorithm can be found in Algorithm 1. Line 2 to line 4 is the data preprocessing step where the RSS measurements in the RFID system are translated into distance information to construct the distance matrix describing the distance between pairwise readers and tags. Line 5 to line 7 refer to the multidimensional scaling step. The absolute positions of the RFID tags can be obtained using the classical metric MDS and the locations of the readers. Line 8 to line 9 correspond to the optional refinement step which solves the MLE problem using the result of MDS at additional computation costs. The refinement is effective when there are sufficient readers in the RFID system. 


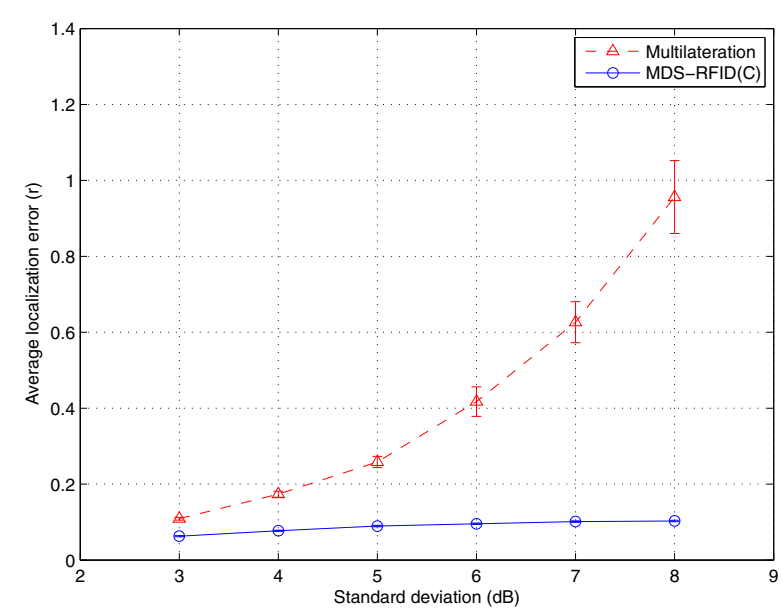

Fig. 3. Performance comparison for multilateration and MDS-RFID(C) when the standard deviation of RSS increases from $3 \mathrm{~dB}$ to $8 \mathrm{~dB}$ and 4 readers are deployed at corners of the area.

\section{Performance Evaluation}

In this section, we simulate the MDS-RFID algorithm and compare it with the multilateration localization scheme [3]. We use least-squares to estimate the positions of the RFID tags for multilateration. In our setup, we choose the communications range of the active RFID tag to be $r=40 \mathrm{~m}$. A total of 30 active RFID tags are deployed randomly in a $10 \mathrm{~m} \times$ $10 \mathrm{~m}$ square area. Therefore, all the tags in the area can communicate with the readers. The path loss exponent in the simulations is chosen to be 2 . The RSS measurements are generated using the log-normal distance path loss model (2). The metric we use to evaluate the performance is the localization error which refers to the distance from the estimated position to the true position of the tag normalized by the communications range $r$. In each set of simulation run, 100 trials with randomly generated topologies are performed and $95 \%$ confidence intervals are plotted in the figures.

\section{A. Effect of the Standard Deviation of RSS}

First, we evaluate the performance of the MDS-RFID algorithm under different standard deviations of RSS in the lognormal path loss model (2). In the simulations, four readers are deployed at the corners of the deployment area and only the lower bound is used for the inter-tag distance estimation. The localization results for multilateration and MDS-RFID $(C)$ when the standard deviation of RSS ranges from $3 \mathrm{~dB}$ to 8 $\mathrm{dB}$ are shown in Fig. 3. From the figure, we can see that the average localization error increases for both algorithms as the standard deviation increases. However, MDS-RFID(C) significantly outperforms multilateration. It can be seen that MDS-RFID(C) is much more robust to measurement noises compared with multilateration. The confidence intervals of MDS-RFID are much smaller than multilateration as well. For standard deviation of $3 \mathrm{~dB}$, MDS-RFID(C) decreases the average localization error by $\frac{0.1089 r-0.0628 r}{0.1089 r}=42.33 \%$ compared with multilateration.

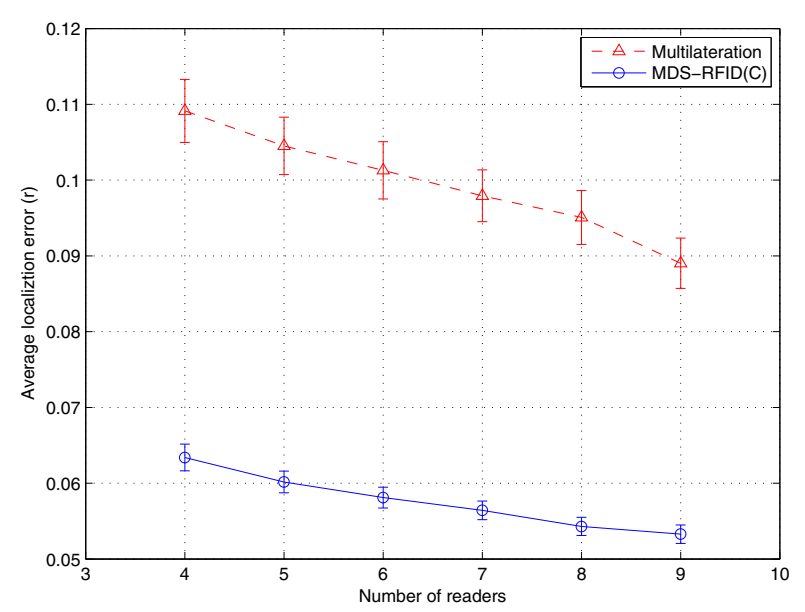

Fig. 4. Performance comparison for multilateration and $\operatorname{MDS}-\operatorname{RFID}(C)$ when the number of readers increases from 4 to 9 and the standard deviation of RSS is $3 \mathrm{~dB}$.

\section{B. Effect of the Number of Readers}

Next, we investigate the impact of the number of readers on the performance. The standard deviation of RSS is set to be $3 \mathrm{~dB}$ which is the typical value in active RFID systems [1]. In addition to the four readers deployed at the corners, the rest of the readers are uniformly placed on the boundary of the deployment area. The average localization error versus the number of readers for MDS-RFID $(C)$ and multilateration is plotted in Fig. 4. From the figure, we can see that the average localization error for both algorithms decreases as the number of readers increases. However, the reasons are different. For multilateration, it is because more tag-to-reader distance measurements are available to increase accuracy. For MDS-RFID(C), it is mainly due to the better estimate of the inter-tag distances using more tag-to-reader distances.

\section{Performance of Refinement}

Finally, we compare the performance between MDS$\operatorname{RFID}(\mathrm{C})$ and $\operatorname{MDS}-\operatorname{RFID}(\mathrm{C}, \mathrm{R})$ with an additional refinement. MDS-RFID $(C, R)$ uses the localization result of MDSRFID(C) as the initial point. The simulation results for different RSS standard deviation is shown in Figure 5. From the figure, we can find that the refinement does not always improve the result of MDS. It even decreases the accuracy when the standard deviation is greater than 4 . This result is not surprising because only four readers are deployed in our simulations. Similar to multilateration but unlike MDS, MLE in the refinement only uses the tag-to-reader distance information. When there are only a few readers in the RFID system, the scarcity of the tag-to-reader distance information limits the performance of MLE.

The simulation results for different number of readers are shown in Fig. 6. It can be seen that $\operatorname{MDS}-\operatorname{RFID}(\mathrm{C}, \mathrm{R})$ improves significantly as the number of readers increases. The refinement can reduce the average localization error by almost a half when the number of readers is 9 . The reason is the same as above, the advantage of the refinement is significant only when there are enough tag-to-reader distance information. 


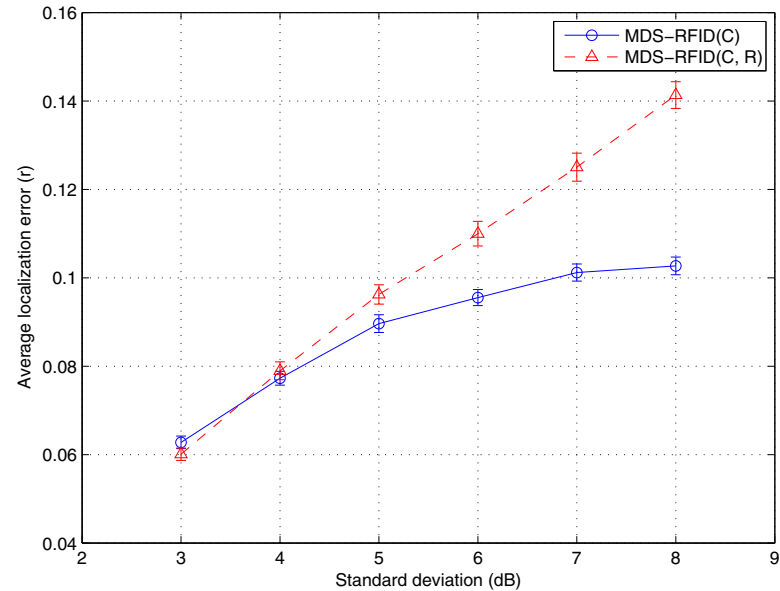

Fig. 5. Performance comparison for MDS-RFID(C) and MDS-RFID(C, R) when the standard deviation of RSS varies from $3 \mathrm{~dB}$ to $8 \mathrm{~dB}$ and 4 readers are deployed at corners of the area.

\section{CONCLUSION}

In this paper, we proposed a novel localization approach in RFID systems based on multidimensional scaling called the MDS-RFID algorithm. It consists of data pre-processing step, multidimensional scaling, and an optional refinement step. In the data pre-processing step, we used the log-normal path loss model to infer the tag-to-reader distances from the RSS measurements, and then estimated the inter-tag distances by the triangular method. Then, classical MDS algorithms can be applied to obtain a coarse localization result. MLE is used to calculate better estimated positions using the result produced by MDS as the initial point in the refinement at the expense of more computations. Simulation results showed that the MDS-RFID algorithm can significantly outperform existing multilateration-based methods. Unlike the LANDMARC system which requires the deployment of reference tags, MDSRFID only needs a few readers. Future work includes applying ordinal MDS and extending the MDS-RFID algorithm to large scale systems.

\section{ACKNOWLEDGMENT}

This research is supported by the Natural Sciences and Engineering Research Council (NSERC) of Canada under grant number 364962-08.

\section{REFERENCES}

[1] R. Want, "An introduction to RFID technology," IEEE Pervasive Computing, vol. 5, no. 1 , pp. 25 - 33, Jan.-Mar. 2006.

[2] M. Bouet and A. dos Santos, "RFID tags: Positioning principles and localization techniques," in Proc. of Wireless Days, Dubai, Nov 2008.

[3] J. Hightower, R. Want, and G. Borriello, "SpotON: An indoor 3D location sensing technology based on RF signal strength," University of Washington, Department of Computer Science and Engineering, Seattle, WA, Tech. Rep., Feb. 2000.

[4] A. Stelzer, K. Pourvoyeur, and A. Fischer, "Concept and application of LPM - A novel 3-D local position measurement system," IEEE Trans. on Microwave Theory and Techniques, vol. 52, no. 12, pp. 2664 - 2669, Dec. 2004.

[5] T. Bechteler and H. Yenigun, "2-D localization and identification based on SAW ID-tags at $2.5 \mathrm{GHz}$," IEEE Trans. on Microwave Theory and Techniques, vol. 51, no. 5, pp. 1584 - 1590, May 2003.

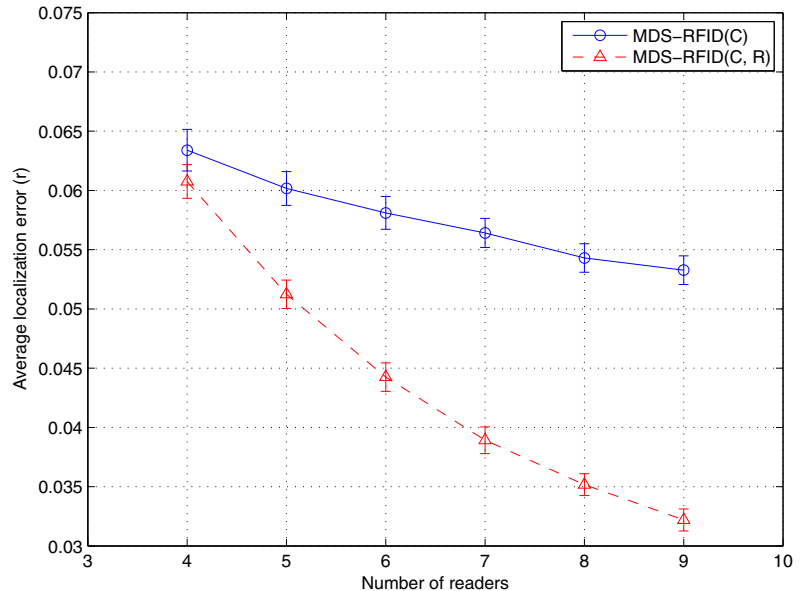

Fig. 6. Performance comparison for $\operatorname{MDS}-\operatorname{RFID}(\mathrm{C})$ and $\operatorname{MDS}-\operatorname{RFID}(\mathrm{C}, \mathrm{R})$ when the number of readers varies from 4 to 9 and the standard deviation of RSS is $3 \mathrm{~dB}$.

[6] C. Hekimian-Williams, B. Grant, X. Liu, Z. Zhang, and P. Kumar, "Accurate localization of RFID tags using phase difference," in Proc. of IEEE Int'l Conf. on RFID, Orlando, FL, Apr 2010.

[7] L. M. Ni, Y. Liu, Y. C. Lau, and A. P. Patil, "LANDMARC: Indoor location sensing using active RFID," ACM/Springer Wireless Networks, vol. 10, pp. 701-710, Nov. 2004.

[8] X. Huang, R. Janaswamy, and A. Ganz, "Scout: Outdoor localization using active RFID technology," in Proc. of BROADNETS, San Jose, CA, Oct 2006.

[9] A. Bekkali, H. Sanson, and M. Matsumoto, "RFID indoor positioning based on probabilistic RFID map and Kalman filtering," in Proc. of IEEE WiMOB, White Plains, NY, Oct 2007.

[10] T. Wada, N. Uchitomi, Y. Ota, T. Hori, K. Mutsuura, and H. Okada, "A novel localization scheme for passive RFID tags; communication range recognition (CRR)," in Proc. of IEEE ICC, Dresden, Germany, June 2009.

[11] A. Almaaitah, K. Ali, H. S. Hassanein, and M. Ibnkahla, "3D passive tag localization schemes for indoor RFID applications," in Proc. of IEEE ICC, Cape Town, South Africa, May 2010.

[12] G. Jin, X. Lu, and M. Park, "An indoor localization mechanism using active RFID tag," in Proc. of IEEE Int'l Conf. on Sensor Networks, Ubiquitous, and Trustworthy Computing, Taichung, Taiwan, Jun. 2006.

[13] S. Jain, A. Sabharwal, and S. Chandra, "An improvised localization scheme using active RFID for accurate tracking in smart homes," in Proc. of Int'l Conf. on Computer Modelling and Simulation, Cambridge, UK, Mar. 2010.

[14] I. Borg and P. Groenen, Modern Multidimensional Scaling: Theory and Applications, 2nd ed. New York: Springer-Verlag, 2005.

[15] Y. Shang, W. Ruml, and Y. Zhang, "Localization from mere connectivity," in Proc. of ACM MobiHoc, Annapolis, MD, Jun. 2003.

[16] Y. Shang and W. Ruml, "Improved MDS-based localization," in Proc. of IEEE Infocom, Hong Kong, China, Mar. 2004.

[17] Y. Shang, W. Ruml, Y. Zhang, and M. Fromherz, "Localization from connectivity in sensor networks," IEEE Trans. on Parallel and Distributed Systems, vol. 15, no. 10, pp. 1-14, Oct. 2004.

[18] V. Vivekanandan and V. W. S. Wong, "Ordinal MDS-based localisation for wireless sensor networks," in Proc. of IEEE VTC-Fall, Montreal, Quebec, Canada, Sept. 2006.

[19] T. S. Rappaport, Wireless Communications: Principles and Practice, 2nd ed. Prentice Hall, 2002.

[20] P. Groenen and M. Van de Velden, "Multidimensional scaling," in Econometric Institute Report EI 2004-15, April 2004.

[21] D. Lay, Linear Algebra and Its Applications, 3rd ed. Addison Wesley, 2003.

[22] J. Bachrach and C. Taylor, Handbook of Sensor Networks: Algorithms and Architectures. Wiley, 2005, ch. Localization in Sensor Networks, pp. 299-304. 